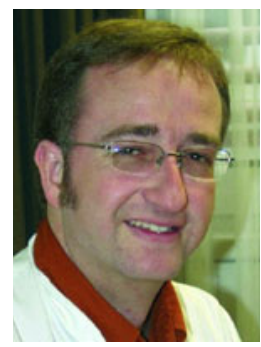

Thomas Wobrock

\title{
Schizophrenie und Sucht
}

\author{
Thomas Wobrock, Frank-Gerald Pajonk, Roberto D’Amelio, Peter Falkai
}

Klinik für Psychiatrie und Psychotherapie, Universitätsklinikum des Saarlandes, Homburg/Saar (Direktor: Prof. Dr. med. P. Falkai)

psychoneuro 2005; 31 (9): 433-440

$\mathrm{D}$ ie Schizophrenie ist eine komplexe Erkrankung, die wesentliche Bereiche des seelischen Erlebens beeinträchtigt und durch ein charakteristisches Störungsmuster in den Bereichen Denken, Wahrnehmung, Ichfunktionen, Affektivität, Antrieb und Psychomotorik gekennzeichnet ist. Mit einer Lebenszeitprävalenz weltweit zwischen 0,5 bis 1,6\% und dem Auftreten erheblicher sozialer Beeinträchtigungen bereits vor dem Auftreten des Vollbildes der Erkrankung stellt die Behandlung der Schizophrenie eine große Herausforderung für unser Gesundheitssystem dar. Noch häufigere Prävalenzraten finden sich für Suchterkrankungen mit einer Lebenszeitprävalenz für Alkoholmissbrauch/-abhängigkeit von $13,5 \%$ und Drogenmissbrauch von $6,1 \%$ in der Gesamtbevölkerung (31). Das Zusammentreffen beider Erkrankungen ist mit einer deutlich schlechteren Prognose behaftet und erschwert nicht selten die Behandlung erheblich. In diesem Beitrag sollen Epidemiologie, Erklärungsversuche der hohen Komorbidität sowie pharmakologische, psychotherapeutische und psychosoziale Behandlungsstrategien dargestellt und erste Empfehlungen abgegeben werden.

\section{- Epidemiologie}

Psychotische Störungen (z.B. schizophrene Psychosen) und Drogen- oder Substanzabhängigkeit bzw. -missbrauch kommen häufig gemeinsam vor. Die Angaben zur Häufigkeit eines Alkohol- oder Drogenmissbrauchs bei Patienten mit endogenen Psychosen variieren er-

Bei etwa 15-65\% aller schizophrenen Patienten liegt gleichzeitig ein komorbider Substanzmissbrauch bzw. eine Substanzabhängigkeit vor. Ein einheitliches Erklärungsmodell für diese hohe Komorbidität besteht nicht. Bevorzugt diskutiert werden derzeit neurobiologische Gemeinsamkeiten beider Krankheitsbilder, eine höhere Sensitivität für psychotrope Substanzen bei schizophrenen Patienten, Drogenkonsum (z.B. Cannabis) als Risikofaktor für die Entwicklung und Aufrechterhaltung der Psychose und prämorbide gemeinsame Persönlichkeitsmerkmale bei Doppeldiagnosepatienten. Trotz der hohen Prävalenz dieser Komorbidität und ihrer hohen klinischen Relevanz durch den schlechteren Krankheitsverlaufs bei persistierendem Drogenkonsum liegen bisher nur wenige Therapiestudien bei dieser Patientengruppe vor. In diesem Beitrag werden die derzeit verfügbaren pharmakologischen und psychotherapeutischen Behandlungsstrategien im Rahmen eines integrativen Behandlungsansatzes dargestellt.

heblich, nach Übersichtsarbeiten liegen die Prävalenzraten zwischen 15\% und 65\% (25). Dabei ist Cannabis in der Regel die häufigste illegale Droge und damit nach Alkohol und Tabak die häufigste missbräuchlich eingenommene Substanz. In Tabelle 1 werden die gefundenen Prävalenzraten für den komorbiden Substanzkonsum in verschiedenen Querschnittserhebungen bei schizophrenen Patienten dargestellt.

Die Unterschiede in den Prävalenzraten sind wesentlich durch methodische Faktoren bedingt. Von besonderer Bedeutung ist dabei neben der Stichprobenzusammensetzung, insbesondere was die Altersverteilung der Patienten und den Anteil chronisch Kranker betrifft, auch die Erfassung des Substanzmissbrauchs, die von globaler klinischer Einschätzung über standardisierte Befragungen bis zur Anwendung toxikologischer Untersuchungen reicht. So finden sich die höchsten Prävalenzraten häufig bei prospektiven Untersuchungen mit einem hohen Anteil an Erstmanifestationen, bei denen der Substanzmissbrauch toxikologisch erfasst wurde (9). Nach klini- schem Eindruck scheint das Ausmaß dieser Komorbidität zuzunehmen, selbst wenn man eine verbesserte Diagnostik und Problemsensibilisierung berücksichtigt. Der einfache Zugang zu Alkohol und Cannabis, insbesondere für junge Menschen, wird nicht zuletzt deswegen als gesellschaftliches Problem gesehen und notwendige Präventionsmaßnahmen als gesellschaftliche Herausforderung betrachtet.

\section{Modelle der Komorbidität}

Erklärungsmodelle für die hohen Prävalenzraten von komorbidem Substanzkonsum bei schizophrenen Psychosen resultieren aus klinischen Beobachtungen und theoretischen Überlegungen, eine wissenschaftliche Absicherung eines bestimmten Modells gelang bisher nicht. Im Wesentlichen werden aktuell vier differente Konzepte unterschieden (14, 18, 27):

- Die Substanzstörung ist Folge der schizophrenen Psychose (sekundäre Suchtentwicklung), wobei am häufigsten das Modell der Selbstmedikation vertreten wird 


\section{Tab. 1 Häufigkeit von Substanzkonsum bei schizophrenen Patienten (Auswahl von Querschnittsstudien)}

\begin{tabular}{|c|c|c|c|c|}
\hline Diagnosen & $\begin{array}{l}\text { Stichproben- } \\
\text { größe }\end{array}$ & Setting & Substanz und Häufigkeit & Literatur \\
\hline $\begin{array}{l}\text { Schizophrenie, schizoaffektive } \\
\text { Psychose, schizophreniforme Psychose }\end{array}$ & 83 & stationär & $\begin{array}{l}\text { Irgendeine Substanz 48\% } \\
\text { THC } 27 \% \\
\text { Alkohol } 25 \% \\
\text { Kokain } 17 \% \\
\text { Stimul./Halluz. } 12 \%\end{array}$ & Dixon et al., 1991 \\
\hline $\begin{array}{l}\text { Schizophrenie und „related } \\
\text { disorders“ (Ersterkrankungen) }\end{array}$ & 93 & stationär & $\begin{array}{l}\text { THC 26\% } \\
\text { Alkoholmissbrauch 9\% } \\
\text { Kokain/XTC 2\% }\end{array}$ & Linszen et al., 1994 \\
\hline Schizophrenie & 139 & stationär & $\begin{array}{l}\text { Alkohol 35\% } \\
\text { THC 19\% }\end{array}$ & Fischer et al., 1996 \\
\hline $\begin{array}{l}\text { Schizophrenie und paranoide } \\
\text { Störungen (Ersterkrankungen) }\end{array}$ & 232 & stationär & $\begin{array}{l}\text { Alkohol 24\% } \\
\text { THC 13\% } \\
\text { Halluzinogene 5\% } \\
\text { Kokain 5\% }\end{array}$ & Hambrecht u. Häfner, 1996 \\
\hline $\begin{array}{l}\text { Schizophrenie und schizophreniforme } \\
\text { Psychose }\end{array}$ & 468 & stationär & $\begin{array}{l}\text { THC 11\% } \\
\text { (übrige Drogen nicht mitgeteilt) }\end{array}$ & Caspari, 1998 \\
\hline $\begin{array}{l}\text { Schizophrenie, schizoaffektive Psychose } \\
\text { (Ersterkrankungen) }\end{array}$ & 89 & stationär & $\begin{array}{l}\text { irgendeine Substanz 36\% } \\
\text { THC 25\% } \\
\text { Amphetamine 5\% } \\
\text { (Alkohol nicht mitgeteilt) }\end{array}$ & Sittinger et al., 2000 \\
\hline Schizophrenie & 125 & stationär & $\begin{array}{l}\text { THC-Konsum } 43 \% \\
\text { Halluzinogene 19\% } \\
\text { Stimulantien 18\% } \\
\text { Opiate } 8 \% \\
\text { Alkoholmissbrauch } 27 \%\end{array}$ & Bersani et al., 2002 \\
\hline Schizophrenie & 85 & stationär & $\begin{array}{l}\text { Lebenszeitprävalenz: } \\
\text { THC 26\% } \\
\text { Alkohol 53\% } \\
\text { Kokain 34\% } \\
\text { Amphetamine 17\% } \\
\text { Halluzinogene 9\% }\end{array}$ & Mueser et al., 1992 \\
\hline $\begin{array}{l}\text { Schizophrenie, schizoaffektive Psychose, } \\
\text { schizotype Persönlichkeitsstörung }\end{array}$ & 82 & ambulant & $\begin{array}{l}\text { signifikanter Gebrauch für } \\
\text { irgendeine Substanz 58\% } \\
\text { THC 31\% } \\
\text { Alkohol 46\% }\end{array}$ & Test et al., 1989 \\
\hline $\begin{array}{l}\text { Schizophrenie, Daten aus populations- } \\
\text { basierter Studie (ECA-Studie) }\end{array}$ & 304 & $\begin{array}{l}\text { allgemeine } \\
\text { Stichprobe }\end{array}$ & $\begin{array}{l}\text { Alkohol 34\% } \\
\text { Drogen 28\% }\end{array}$ & Regier et al., 1990 \\
\hline
\end{tabular}

- Schizophrene Psychosen sind durch den Substanzkonsum entscheidend mitverursacht bzw. werden durch den Substanzkonsum induziert (sekundäre Psychoseentwicklung)

- Schizophrene Psychosen und Substanzstörungen haben eine gemeinsame neurobiologische bzw. genetische Ursache z.B. im Sinne einer Dysfunktion des dopaminergen Systems

- Schizophrene Psychosen und Substanzstörung halten sich gegenseitig aufrecht (bidirektionales Modell) oder sind über verschiedene Mechanismen mitei- nander verknüpft (integratives Modell).

Im Modell der sekundären Suchtentwicklung sind für die Selbstmedikationshypothese (21) bisher keine überzeugende Belege vorgelegt worden. Unterschiede in der Psychopathologie zwischen schizophrenen Patienten mit und ohne komorbidem Substanzkonsum konnten bisher nicht konsistent gefunden werden, und es zeigte sich keine Präferenz bestimmter Substanzen (wie z.B. Amphetamine oder Kokain) bei bestimmter Symptomatikausprägung (z.B. Negativsymptomatik) (9).
Als Beleg für das Modell der sekundären Psychoseentwicklung am Beispiel des Cannabiskonsums werden epidemiologische, populationsbasierte Verlaufsuntersuchungen herangezogen (siehe Tab. 2), in denen sich zumeist dosisabhängig der Cannabiskonsum als bedeutender Risikofaktor für eine spätere Psychose erwies wie beispielsweise in der bereits 1987 veröffentlichen Verlaufsstudie an schwedischen Rekruten (1). Einschränkend ist allerdings zu werten, dass z.B. in einer Studie an Ersterkrankten nur rund ein Drittel der Patienten einen Drogenkonsum vor Auftreten der ers- 
ten psychotischen Episode aufwiesen (19).

Das Modell der gemeinsamen ätiologischen Faktoren berücksichtigt mögliche gemeinsame biochemische, genetische und umweltbedingte Grundlagen. Die Häufung von Suchterkrankungen in den Familien schizophrener Patienten mit komorbidem Drogenkonsum mag für eine genetische Komponente sprechen, bislang erbrachten Zwillingsstudien, genetische Kopplungsanalysen oder Assoziationsunterschungen aber keine überzeugenden positiven Resultate (7). In Abbildung 1 werden gemeinsame neurobiologische Grundlagen der Sucht- und Psychoseentwicklung dargestellt, welche sich vor allem in einer Dysbalance des dopaminergen Systems ausdrücken (10). Daneben existieren noch ergänzende Modelle, z.B. das gehäufte Vorkommen gemeinsamer Merkmale wie einer antisozialen Persönlichkeitsstruktur (27) oder das Konzept der gemeinsamen Grundlage einer Störung der Affektregulation (7). Favorisiert wird derzeit von einigen Autoren am ehesten das Modell der antisozialen Persönlichkeitsstörung (APS) (aus dem Modell der gemeinsamen Faktoren) und das Supersensitivitätsmodell (aus dem Modell der sekundären Substanzstörung) (28). Patienten nach dem APSModell haben eine ausgeprägte Substanzstörung bei hoher psychischer Belastung. Sie lassen sich charakterisieren durch einen früheren Beginn der Substanzstörung, den Gebrauch einer hohen Anzahl von Suchtmitteln, einer stärkeren körperlichen Abhängigkeit, einer häufigeren positiven Familienanamnese für Substanzkonsum, einem früheren Erstmanifestationsalter der psychotischen Erkrankung, einer schlechteren prämorbiden sozialen Funktion, mehr psychiatrischen Symptomen, einer höheren Neigung zu Aggressivität und einer ungünstigeren Prognose. Diese Patienten brauchen eine umfangreiche Betreuung z.B. durch stationäre Therapie, aufsuchende Strategien und dichtes Monitoring (11). Die Patienten mit erhöhter Sensitivität für die Wirkung, aber nicht die Ab- hängigkeit von psychotropen Substanzen („Supersensitivität“), die nur gelegentlich Drogen konsumieren, eine niedrigere psychische Belastung und eine günstigere Prognose aufweisen, scheinen dagegen von psychoedukativen Maßnahmen und einer Behandlung im ambulanten Setting zu profitieren (11). Aufgrund der widersprüchlichen Studienbefunde bei insgesamt heterogener Gruppe der Doppeldiagnosepatienten, erscheint es plausibel, mehrere Entstehungsmöglichkeiten anzunehmen.

Ein integratives Modell (18) trägt dieser komplexen Situation Rechnung und erklärt, je nach unterschiedlicher Gewichtung im Einzelfall, z.B. den Substanzmissbrauch als primär ungünstigen Copingversuch bei vulnerablen jungen Menschen mit Prodromalsymptomen einer Schizophrenie, der seinerseits wieder den Ausbruch der Psychose begünstigt. Dieses Modell weist Ähnlichkeiten mit dem Vulnerabilitätsmodell zur Entstehung schizophrener Psychosen auf, indem z.B. regelmäßiger Cannabiskonsum als (unabhängiger)

\section{Tab. 2 Prospektive epidemiologische Studien zum Risiko von Cannabiskonsumenten für Schizophrenie}

\begin{tabular}{|c|c|c|c|c|c|c|}
\hline Beschreibung der Stichprobe & $\begin{array}{l}\text { Stich- } \\
\text { proben- } \\
\text { größe }\end{array}$ & $\begin{array}{l}\text { Unter- } \\
\text { suchungs- } \\
\text { jahr }\end{array}$ & $\begin{array}{l}\text { Follow- } \\
\text { up } \\
\text { (Jahre) }\end{array}$ & $\begin{array}{l}\text { Beurteilungs- } \\
\text { kriterium }\end{array}$ & $\begin{array}{l}\text { Relatives Risiko } \\
\text { oder Odds-Ratio }\end{array}$ & Literatur \\
\hline $\begin{array}{l}\text { Kohorte aus Rekruten (Schweden), } \\
\text { Männer, Konsumalter } 18 \text { Jahre }\end{array}$ & 50053 & 1969-1970 & 27 & $\begin{array}{l}\text { stationäre Behandlung } \\
\text { wegen Schizophrenie } \\
\text { (ICD 8/9) }\end{array}$ & $\begin{array}{l}\text { bei über 50maligem } \\
\text { Konsum ca. 6.7, nach } \\
\text { Korrektur für konfun- } \\
\text { dierende Variablen } 3.1\end{array}$ & $\begin{array}{l}\text { Zammit } \\
\text { et al., } 2002\end{array}$ \\
\hline $\begin{array}{l}\text { Musterungskohorten (Draft Board } \\
\text { Assessment), männliche Jugendliche, } \\
\text { Israel, 16-17 Jahre }\end{array}$ & 9724 & $\begin{array}{l}\text { nicht an- } \\
\text { gegeben }\end{array}$ & $4-15$ & $\begin{array}{l}\text { stationäre Behandlung } \\
\text { wegen Schizophrenie } \\
\text { (ICD 9) }\end{array}$ & ca. 2,0 & $\begin{array}{l}\text { Weiser } \\
\text { et al., } 2002\end{array}$ \\
\hline $\begin{array}{l}\text { Populationsbasierte Erhebung } \\
\text { (NEMESIS-Studie, Niederlande), } \\
\text { Männer und Frauen, Konsumalter } \\
\text { 18-64 Jahre }\end{array}$ & 4104 & 1994-1996 & 3 & $\begin{array}{l}\text { psychotische Symptome } \\
\text { anhand der BPRS }\end{array}$ & $\begin{array}{l}\text { ca. } 2,8 \text { für psychotische } \\
\text { Symptome }\end{array}$ & $\begin{array}{l}\text { Van Os } \\
\text { et al., } 2002\end{array}$ \\
\hline $\begin{array}{l}\text { Geburtskohorte (Dunedin-Studie, } \\
\text { Neuseeland), Männer und Frauen, } \\
\text { Konsumalter } 15 \text { Jahre }\end{array}$ & 759 & 1972-1973 & 11 & $\begin{array}{l}\text { Diagnose einer schizo- } \\
\text { phreniformen Störung } \\
\text { nach DSM-IV }\end{array}$ & ca. 4 & $\begin{array}{l}\text { Arsenault } \\
\text { et al., } 2002\end{array}$ \\
\hline $\begin{array}{l}\text { Geburtskohorte, CDHS-Studie, } \\
\text { Männer und Frauen, Neuseeland, } \\
\text { 18-25 Jahre }\end{array}$ & 1055 & 1977 & $\begin{array}{l}\text { (jeweils } 1 \\
\text { Jahr) } 7\end{array}$ & $\begin{array}{l}\text { psychotische Symptome } \\
\text { nach SCL-90 (10 Items) }\end{array}$ & $\begin{array}{l}\text { 2,3-3,3, nach Korrek- } \\
\text { tur 1,6-1,8 für täg- } \\
\text { lichen Konsum, aller- } \\
\text { dings jeweils auf } 1 \text { Jahr } \\
\text { bezogen }\end{array}$ & $\begin{array}{l}\text { Fergusson } \\
\text { et al., } 2005\end{array}$ \\
\hline $\begin{array}{l}\text { populationsbasierte Erhebung } \\
\text { (Stadtbezirke München), Männer } \\
\text { und Frauen, Deutschland, 14-24 Jahre }\end{array}$ & 2437 & 1995 & 4 & $\begin{array}{l}\text { psychotische Symptome } \\
\text { (mind. } 2 \text { von } 15 \text { Psy- } \\
\text { chose-Items des M-CIDI) }\end{array}$ & 2,23 & $\begin{array}{l}\text { Henquet } \\
\text { et al., } 2005\end{array}$ \\
\hline
\end{tabular}




\section{Abb. 1 Gemeinsame neurobiologische Grundlage von Psychose und Sucht}

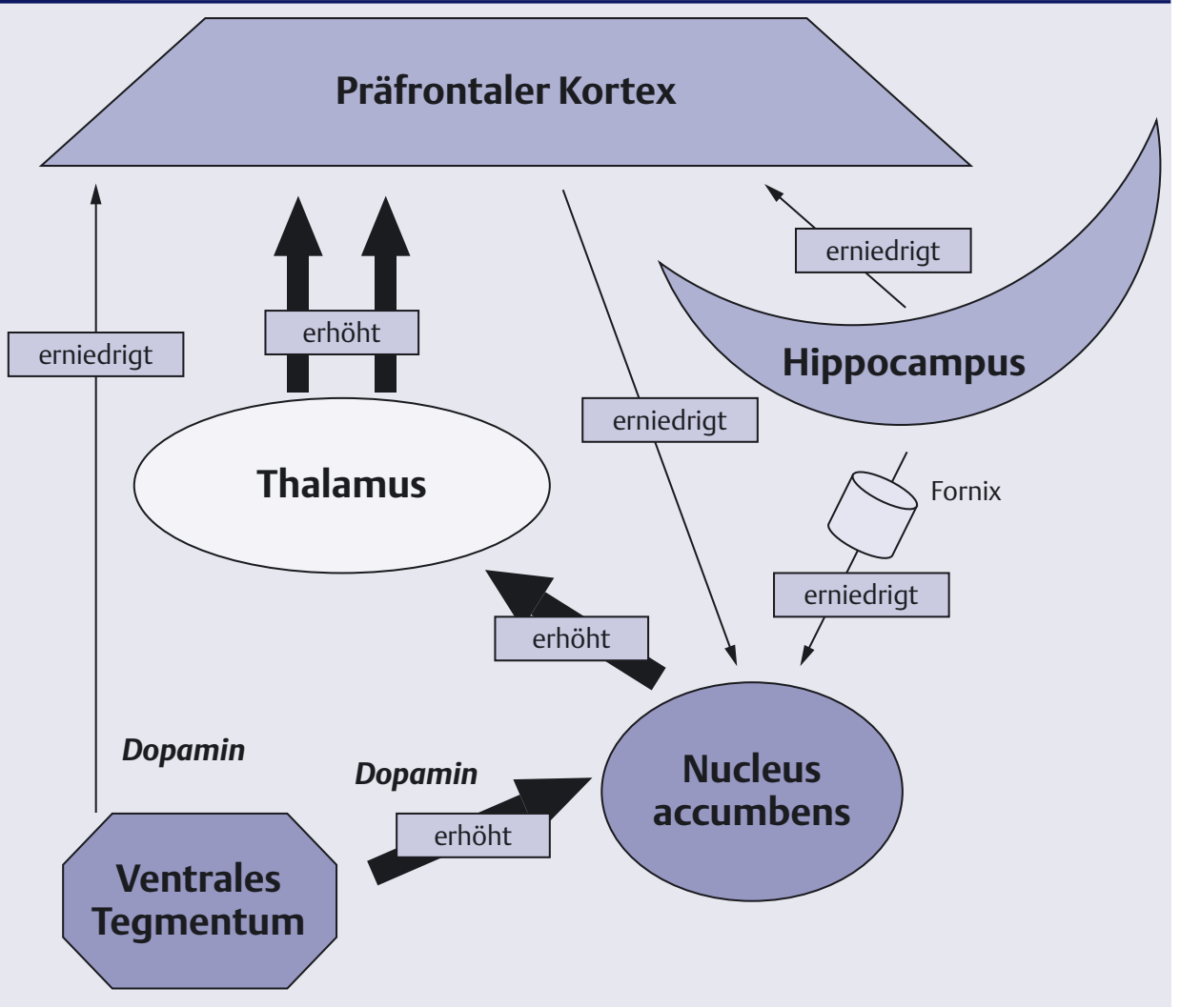

Bei Suchterkrankungen wird eine Dysfunktion im mesolimbischen Belohnungssystem („,reward system“) angenommen. Suchtstoffe führen zu einer verstärkten Dopamin-Freisetzung von Projektionen aus dem ventralen Tegmentums in den Nucleus accumbens (Aktivierung des Belohnungssystems). Chronischer Konsum führt zu neuroadaptiven Prozessen („Sensitivierung“) mit sekundärer Minderung der kortikalen präfrontalen Kontrolle über dieses Belohnungssystem. Bei der Schizophrenie werden eine dopaminerge Hyperaktivität im mesolimbischen System und eine Minderaktivität im mesokortikalen System angenommen. Eine primär verminderte glutamaterge Projektion vom präfrontalen Kortex zum Nucleus accumbens soll in diesem Kerngebiet ebenfalls eine erhöhte dopaminerge Aktivität zur Folge haben. Bei beiden Erkrankungen führt somit eine Dysbalance dopaminerger Systeme zugunsten einer Hyperaktivität in subkortikalen Strukturen zur Anfälligkeit für Störungen der Verarbeitung bestimmter Stimuli (z.B. „Reizüberflutung“) (modifiziert nach 10, 17).

Risikofaktor für das spätere Auftreten der schizophrenen Psychose in einem grundsätzlich multifaktoriellen Bedingungsgefüge angesehen wird, wobei von einer komplexen Interaktion mit anderen Vulnerabilitätsfaktoren (z.B. genetischen Mechanismen oder neurostrukturellen Abweichungen oder auch psychosozialen Faktoren) auszugehen ist. In einer Untersuchung wiesen schizophrene Patienten mit komorbidem Substanzkonsum weniger hirnmorphologische Auffälligkeiten auf als schizophrene Patienten ohne Drogenmissbrauch (42). Dies weist darauf hin, dass eher der zusätzliche Drogenkon- sum bei den Doppeldiagnosepatienten die Schwelle für die Entwicklung einer Psychose senkt als Störungen der Hirnentwicklung.

Für diese Interpretation sprechen auch neuere größere sozioepidemiologische Erhebungen, in denen im Längsschnittverlauf Cannabiskonsum als ein Faktor gesehen wurde, welcher dosisabhängig, das Risiko an einer Schizophrenie zu erkranken, erhöht. Schwedische Rekruten, die mehr als 50 mal Haschisch geraucht hatten, wiesen ein um 6,7-fach erhöhtes Risiko auf, später an einer Schizophrenie zu erkranken (43). Insgesamt wurde aus diesen Daten ge- folgert, dass Cannabiskonsum das Risiko einer schizophrenen Erkrankung um 30\% steigert (bzw. dass 13\% der schizophrenen Krankheiten verhindert werden könnten, wenn es gelänge, den Cannabismissbrauch in der Bevölkerung einzudämmen). Allerdings können bei Personen mit einer Krankheitsdisposition vermutlich schon relativ niedrige Dosen Cannabis eine schizophrene Psychose triggern, wenn der Drogenkonsum in einem vulnerablen Alter stattfindet (9). Dafür, dass Drogenkonsum das Risiko für eine spätere Schizophrenie in relevantem Ausmaß vermutlich nur bei vorbestehender Vulnerabilität erhöht, spricht die Tatsache, dass zwar der Cannabiskonsum in der Bevölkerung gerade unter Jugendlichen und jungen Erwachsenen in den letzten Jahrzehnten (22), aber nicht die Prävalenz der Schizophrenie in den westlichen Ländern angestiegen ist.

Trotz der hohen klinischen Relevanz dieser Doppeldiagnose sind effiziente Therapiestrategien insbesondere im Hinblick auf die Pharmakotherapie bisher kaum entwickelt worden. Ein Grund hierfür könnte sein, dass dieses Patientenklientel nur schwierig in klinischen Studien zu betreuen ist. In pharmakotherapeutischen Studien zur Wirksamkeit, Sicherheit und Effektivität von Antipsychotika wird diese Patientengruppe oft ausgeschlossen.

\section{Grundlagen der Therapie}

In der stationären, aber auch ambulanten Versorgung sind Patienten mit schizophrener Erkrankung und gleichzeitigem komorbidem Substanzkonsum häufig. Deswegen sollte bei schizophrenen Patienten gezielt danach exploriert werden. Bei klinischem Verdacht auf das Vorliegen eines zusätzlichen Substanzgebrauches sollte, wenn möglich, eine toxikologische Untersuchung erfolgen. Da ein persistierender Substanzkonsum die Prognose der schizophrenen Erkrankung ungünstig beeinflusst, zu verminderter Compliance, zu häufigeren Rezidiven, einer höheren Rate an tardiven Dyskinesien, höherer Belastung der Angehörigen, reduziertem sozioökonomischen Status und mehr forensischen Komplikationen führt, ist für diese 
Patienten mit Doppeldiagnose ein spezieller Behandlungsrahmen zu empfehlen. Dieser sollte eine integrierte Behandlung beider Erkrankungen einschließen und auch, wenn verfügbar, ein spezialisiertes Behandlungsteam in die Therapie einbinden (14). Ziele und Behandlungsansätze sind eine Besserung der psychotischen Symptomatik (Positiv- und Negativsymptomatik, Depressivität, soziale Ängste), ein frühzeitiges Erkennen und Behandeln extrapyramidal-motorischer Symptome, eine Aufklärung über die negativen Folgen des Substanzkonsums, beständige Motivation, den Substanzkonsum einzuschränken, eine Abklärung und Behandlung evtl. organischer Folgeerkrankungen des Substanzkonsums und die Entwicklung von Bewältigungsstrategien in rückfallgefährdenden Situationen. Ein integrativer Therapieansatz ist auch deshalb zu empfehlen, da die Behandlung dieser Patientengruppe in voneinander getrennten Institutionen mit Stabilisierung und Behandlung der schizophrenen Symptomatik in einer Psychiatrischen Klinik und paralleler oder nachfolgender Behandlung der Substanzstörung in einer suchtspezifischen Einrichtung weniger wirksam und effektiv ist. Neben der fehlenden Kontinuität in der Betreuung mit damit einhergehendem mangelndem Patientenvertrauen, Informationsdefiziten und Schwierigkeiten in der Zusammenarbeit sind schizophrene Patienten in einem suchtzentrierten Setting mit dem dort vertretenen eher konfrontativen Interaktionsstil, der geforderten hohen Eigenverantwortlichkeit und der beständigen Selbstmotivation häufig überfordert. Dies gilt umso mehr, je ausgeprägter die schizophrene Symptomatik einschließlich kognitiver Defizite ist.

Im Rahmen eines integrativen Ansatzes erfolgt das therapeutische Angebot aus einer Hand und kann entsprechend dem Konzept der niederschwelligen, langzeitlich angelegten Behandlung individuell auf den aktuellen Gesundheitszustand, die aktuelle Motivation, die kognitiven und psychosozialen Ressourcen und die derzeitigen Bedürfnisse des Patienten abgestimmt werden. Da- bei sollte die Behandlung unter Erstellung eines persönlichen Störungs- und Veränderungsmodells unter Berücksichtigung der Biografie und der aktuellen Lebensumstände des Patienten erfolgen.

\section{Pharmakotherapeutische Strategien}

Die pharmakologische Behandlung von Patienten mit der Komorbidität von Schizophrenie und Substanzstörung sollte generell in einen Gesamtbehandlungsplan eingebettet sein und im Rahmen eines integrativen Therapieansatzes erfolgen. Während in einigen Übersichtsarbeiten konventionelle Antipsychotika (z.B. mit zusätzlicher $\mathrm{D}_{1}$-agonistischer Wirkung) mit dem Schwerpunkt auf der Depotapplikation hervorgehoben werden (36) oder trizyklischen Antidepressiva und Benzodiazepinen ein höherer Stellenwert zugemessen wird (41), werden in anderen Reviews zunehmend neuere Antipsychotika als Behandlungsalternative genannt (29) und hierfür Hypothesen entwickelt (30). Aufgrund des komplexen Zusammenhangs beider Störungen, der sehr interindividuell unterschiedlichen Ausprägung beider Krankheitsbilder und der differenten ätiologischen Krankheitsmodelle dieser Komorbidität sind derzeit keine einheitlichen pharmakotherapeutischen Empfehlungen verfügbar. Meta-Analysen oder systematische Reviews mit quantitativer Studienauswertung liegen nicht vor.

Prinzipiell können mehrere wesentliche Behandlungsabschnitte und -schwerpunkte bei Patienten mit der Doppeldiagnose einer schizophrenen Störung und eines Substanzkonsums unterteilt werden, die einer pharmakologischen Therapie bedürfen (modifiziert nach 36 ), so die Therapie von Intoxikationen und Entzugssymptomen, Therapie von psychiatrischen Symptomkonstellationen z.B. schizophrenen oder depressiven Symptomen, Rückfallverhütung bei der schizophrenen Erkrankung, Management unerwünschter Begleitwirkungen der antipsychotischen Medikation und eine Reduktion/Rückfallverhütung in Bezug auf den Substanzkonsum.
Bei der Therapie von Intoxikationen und Entzugssymptomen ist nach den üblichen Standards zu verfahren, spezielle Behandlungsempfehlungen für Patienten mit Schizophrenie liegen nicht vor (36).

Bei der medikamentösen Therapie der schizophrenen Symptome der Doppeldiagnosepatienten sollte eine mögliche Verstärkung der anticholinergen Begleitkomponente der verordneten Antipsychotika (z.B. Blasenstörung, Tachykardie, kognitive Beeinträchtigung) durch den zusätzlichen Konsum ebenfalls anticholinerg wirkender Drogen beachtet werden. Aufgrund der verringerten Compliance werden in der Erhaltungstherapie eher Depot-Neuroleptika wie beispielsweise Risperidon Microspheres und Flupenthixoldecanoat empfohlen. Bei den Atypika erwies sich bei dieser Patientengruppe Olanzapin in offenen, prospektiven Studien als wirksam und zeigte einen Vorteil nach Umstellung von konventionellen Neuroleptika. Ansonsten liegen bei den atypischen Antipsychotika mit Clozapin bislang die meisten Erfahrungen für die Behandlung von schizophrenen Patienten mit komorbidem Drogenkonsum vor (44). In der Rezidivprophylaxe zeigte sich unter Clozapintherapie kein Unterschied zwischen schizophrenen Patienten mit und ohne Drogenkonsum.

In offenen Studien, Fallserien und Kasuistiken führten Behandlungen mit Clozapin, Olanzapin, Risperidon, und in einer kürzlich veröffentlichten offenen Verlaufsstudie auch mit Aripiprazol (5) zur Reduktion des Substanzkonsums bei begleitender Kokainabhängigkeit und mit Flupentixoldecanoat bei komorbider Alkoholabhängigkeit (37). Auch die zusätzliche Gabe von Desipramin und Imipramin zur neuroleptischen Erhaltungstherapie verringerte das Suchtverlangen und den Kokainkonsum $(33,34)$, so dass der additive Einsatz von Antidepressiva zur Reduktion des Cravings zumindest bei komorbidem Kokainmissbrauch zu erwägen ist. In der bisher einzigen prospektiven, doppelblinden, randomisierten, kontrollierten Studie mit Antipsychotika zeigte Olanzapin keine Überlegenheit bezüglich Craving, Psychopathologie und Ausmaß des Substanz- 
konsums gegenüber Haloperidol bei kokainabhängigen schizophrenen Patienten (32). Bei den Anti-CravingSubstanzen wird Acamprosat bei zusätzlicher Alkoholabhängigkeit empfohlen. Erfahrungen bei schizophrenen Patienten liegen mit Naltrexon und Disulfiram vor, wobei letzteres allerdings aufgrund seiner potenziellen psychoseinduzierenden Eigenschaft nicht generell empfohlen wird.

\section{Psychotherapeutische Interventionen}

Das psychotherapeutische Angebot im Rahmen eines integrativen Ansatzes sollte niederschwellig und langzeitlich angelegt sein, durch einund dasselbe Behandlungsteam erfolgen und individuell auf den aktuellen Gesundheitszustand, die aktuelle Motivation, die kognitiven und psychosozialen Ressourcen und die derzeitigen Bedürfnisse des Patienten abgestimmt werden (14). Dabei sollte die Behandlung eine Erstellung des persönlichen Störungs- und Veränderungsmodells unter Berücksichtigung der Biografie und der aktuellen Lebensumstände des Patienten beinhalten.

Ein integratives Therapieprogramm sollte Maßnahmen zur Behandlung der Schizophrenie (Förderung von Krankheitseinsicht und Compliance bezüglich der regelmäßigen Einnahme von Medikamenten, dem Erkennen von psychotischen Frühwarnsymptomen und dem Rückfallmanagement), zur Behandlung der Abhängigkeitsproblematik (Erkennen und Akzeptieren des Missbrauchs, der Förderung von Abstinenzzuversicht bzw. -motivation, der Rückfallprophylaxe und Rückfallmanagement sowie der Schaffung von „gesunden“ Alternativen zum Substanzmissbrauch), und zur Behandlung des Erkennens und Akzeptierens der Zusammenhänge zwischen Substanzkonsum und Verlauf der schizophrenen Psychose sowie zur Förderung von Lebensqualität einschließen.

Da viele Patienten Schwierigkeiten haben, sich von vornherein auf eine langfristige abstinenzorientierte Therapie einzulassen, wurde hierzu ein gestuftes Vorgehen nach Stabilisierung der schizophrenen Symptomatik analog der traditionel- len suchtspezifischen Therapie mit Kontaktphase, Motivationsphase, aktiver Therapiephase sowie Stabilisierungsphase und eine Aufteilung der Interventionen in diese Stadien vorgeschlagen. Es liegt Evidenz dafür vor, dass mehrdimensionale verhaltenstherapeutische Programme unter Einschluss motivationaler, psychoedukativer und familientherapeutischer Elemente hinsichtlich einer Verbesserung der Rückfallrate, der Positivsymptomatik und der sozialen Funktion der Standardbehandlung überlegen sind (3).

Während für schizophrene Störungen eine Reihe psychoedukativer Manuale vorliegen (z.B. 4), gibt es für Patienten mit Doppeldiagnosen (schizophrene Störung mit komorbidem Substanzkonsum) bisher bereits zwei deutschsprachige Manuale $(11,17)$.

\section{Soziotherapeutische Möglichkeiten}

Eine intensive Betreuung (Intensive Case Management) innerhalb eines integrierten Behandlungsangebots (gleichzeitig für die schizophrene Störung und den Substanzkonsum) ist einer Standardbehandlung überlegen. Im Lebensumfeld der Patienten sollte eine aufsuchende gemeindenahe Behandlung (Assertive Community Treatment) implementiert werden, da diese sowohl hinsichtlich der sozialen Situation und der Lebensqualität als auch einer Verbesserung der Psychopathologie und einer Reduktion des Substanzkonsums Vorteile gegenüber der Standardbehandlung verspricht (13). Rehabilitation und Wohnsituation sollten in ein integriertes Therapieangebot eingebettet sein, wobei ansonsten bei dieser Patientengruppe ähnliche Grundsätze gelten sollten wie bei schizophrenen Patienten ohne Substanzstörung.

\section{Fazit}

Schizophrene Psychosen weisen eine hohe Komorbidität mit einer Substanzstörung insbesondere bei Ersterkrankten auf. Ein einheitliches Modell für die Komorbidität von Substanzgebrauch und schizophrener Störung gibt es nicht. Integrative Modellvorstellungen beziehen verschiedene Faktoren mit ein und gewichten sie im Einzelfall. Der beglei- tende Substanzkonsum schizophrener Patienten führt langfristig zu einem schlechteren Verlauf mit häufigeren psychotischen Rückfällen, persistierenden Symptomen und höherer psychosozialer Beeinträchtigung. Patienten mit dieser Doppeldiagnose benötigen ein spezielles Therapiekonzept mit einem integrativen Behandlungsansatz, welcher beiden Erkrankungen Rechnung trägt. Bei zunehmendem Ausmaß dieses Komorbiditätsproblems stellt die Entwicklung geeigneter medikamentöser und psychotherapeutischer Strategien eine Herausforderung dar. Eine atypische neuroleptische Behandlung und multimodale psychotherapeutische Behandlungsprogramme erscheinen hierbei erfolgsversprechend.

Schizophrenia and substance abuse Comorbid substance abuse or dependence is found in approximately 15$65 \%$ of schizophrenic patients. While there is no generally accepted hypothesis for this frequent comorbidity, the models preferentially discussed include common neurobiological factors of both diseases, an increased sensitivity for psychotropic drugs in schizophrenics, substance use as a risk factor for developing or promoting psychosis and common premorbid personality traits of both diseases. Despite the high prevalence and the association of persisting substance abuse and poor outcome, there are only few controlled studies in dual diagnosis patients available. The nowadays suggested pharmacological and psychological treatment options due to an integrative treatment approach are discussed in this paper.

\section{Key words}

Schizophrenia - substance abuse dual diagnosis - epidemiology treatment

\section{Literatur bei der Redaktion oder} unter www.psychoneuro.info

\section{Korrespondenzadresse:}

Dr. med. Thomas Wobrock

Klinik für Psychiatrie und Psychotherapie Universitätsklinikum des Saarlandes 66421 Homburg/Saar

thomas.wobrock@uniklinik-saarland.de 